\title{
Tendência das taxas de detecção de hanseníase em jovens de 10 a 19 anos de idade nas Regiões de Integração do estado do Pará, Brasil, no período de 2005 a 2014
}

\section{Trend in detection rates of leprosy in youngs from 10 to 19 years old in the Regions of Integration of Pará State, Brazil, from 2005 to 2014}

\author{
Dilma Costa de Oliveira Neves , Cláudia Daniela Tourão Ribeiro', Larissa Ernesto Santos e Santos ${ }^{1}$, Diana da Costa \\ Lobato $^{2}$ \\ ' Centro Universitário do Estado do Pará, Belém, Pará, Brasil \\ 2 Secretaria de Saúde do Estado do Pará, Belém, Pará, Brasil
}

\begin{abstract}
RESUMO
INTRODUÇÃO: A ocorrência de hanseníase em jovens pode representar a fragilidade do controle da doença e a permanência de sua endemicidade. OBJETIVO: Analisar a tendência da taxa de detecção da hanseníase em jovens de 10 a 19 anos de idade, nas Regiões de Integração do estado do Pará, Brasil, no período de 2005 a 2014. MATERIAIS E MÉTODOS: Estudo ecológico exploratório baseado em coleta de dados do Sistema de Informação de Agravos de Notificação armazenados no Departamento de Epidemiologia da Secretaria de Estado de Saúde Pública do Pará. Foram analisadas 7.606 notificações de hanseníase em jovens, considerando as variáveis: sexo, raça/cor, idade, escolaridade, gravidez, município de residência, ano do diagnóstico da doença, forma clínica e classificação operacional. RESULTADOS: As maiores proporções de casos novos ocorreram nas regiões Carajás $(14,23 \%)$ e Metropolitana (14,07\%). As maiores taxas de detecção foram observadas nas regiões: Metropolitana (389,0/100.000), Lago Tucuruí (259,2/100.000) e Carajás (196,7/100.000). As maiores reduções no número de casos novos ocorreram nas regiões Araguaia (69,5\%), Lago Tucuruí (67,9\%) e Baixo Amazonas (65,6\%). Quanto aos perfis clínico e sociodemográfico, os mais prevalentes foram: sexo masculino; raça/cor parda; ensino fundamental incompleto; jovens não grávidas; forma clínica indeterminada; e classificação operacional paucibacilar. Houve superioridade proporcional da forma clínica dimorfa entre as multibacilares, em comparação à proporção da forma indeterminada entre as paucibacilares. CONCLUSÃO: Considera-se que o estado do Pará ainda se encontra distante de uma evolução favorável no controle da hanseníase entre os jovens, por isso são necessárias estratégias que tornem eficazes as ações de controle da doença.
\end{abstract}

Palavras-chave: Hanseníase; Epidemiologia Descritiva; Criança; Adolescente.

\begin{abstract}
INTRODUCTION: The occurrence of leprosy in youngs may represent the weakness of disease control and the permanence of its endemicity. OBJECTIVE: To analyze the tendency of detection rate of leprosy in youngs from 10 to 19 years old in the Regions of Integration of Pará State, Brazil, from 2005 to 2014. MATERIALS AND METHODS: Exploratory ecological study based on data collection from the Information System on Diseases of Compulsory Declaration stored in Department of Epidemiology of Secretaria de Estado de Saúde Pública of Pará State. The study analyzed 7,606 reports of leprosy in youngs, considering the following variables: sex, race/color, age, schooling, pregnancy, municipality of residence, year of disease diagnosis, clinical form and operational classification. RESULTS: The largest proportions of new cases occurred in Carajás (14.23\%) and Metropolitana (14.07\%) regions. The highest detection rates were observed in the regions: Metropolitana (389.0/100,000), Lago Tucuruí $(259.2 / 100,000)$, and Carajás $(196.7 / 100,000)$. The greatest reductions in the number of new cases occurred in Araguaia (69.5\%), Lago Tucuruí (67.9\%), and Baixo Amazonas (65.6\%) regions. Relating to clinical and sociodemographic profiles, the most prevalent were: male gender; race/brown skin; incomplete elementary school; non-pregnant youngs; indeterminate clinical form; and paucibacillary operational classification. There was a proportional superiority of the dimorphic clinical form among the multibacillary comparing to the proportion of the indeterminate form among the paucibacillary. CONCLUSION: Pará State is still considered far from a favorable evolution in the leprosy control among youngs, so it is necessary strategies that make effective actions of disease control.
\end{abstract}

Keywords: Leprosy; Descriptive Epidemiology; Child; Teenager.

\footnotetext{
Correspondência / Correspondence:

Dilma Costa de Oliveira Neves

Trav. Chaco, 2155. Bairro: Marco - CEP: 66093-572 - Belém, Pará, Brasil - Tel.: + 55 (91) 99233-2525

E-mail: dilmaconeves@gmail.com
} 


\section{INTRODUÇÃO}

A hanseníase é uma das doenças mais antigas da humanidade e, apesar da cura por meio da poliquimioterapia, ela ainda constitui um importante problema de saúde pública no Brasil| ${ }^{1,2}$.

A despeito dos esforços empregados, tanto pela Organização Mundial da Saúde (OMS) em países endêmicos como pelo Programa Nacional de Controle da Hanseníase, o número de casos novos vem declinando modestamente em algumas regiões do Brasil e crescendo em outras. Segundo a OMS, em 2014, 213.899 novos casos de hanseníase foram relatados no mundo, e $94 \%$ desses estão concentrados em 13 países endêmicos que são alvos dos programas de combate à doença. A Índia foi o destaque, com 125.785 casos diagnosticados, e o Brasil, com 31.064 casos, foi o segundo país em número de diagnósticos ${ }^{3}$.

Segundo o Ministério da Saúde do Brasil, no ano de 2015, o estado do Pará foi o quarto em número de pessoas com hanseníase, com taxa de detecção geral de casos novos de 35,2 casos/100.000 habitantes. Com taxas acima desse valor, encontram-se os estados do Mato Grosso, Tocantins e Maranhão ${ }^{4}$.

As diferentes formas clínicas da hanseníase dependem da resposta imunológica do hospedeiro frente à presença do bacilo. Tais expressões clínicas vão desde uma resposta imunológica competente a uma menos efetiva, ou seja, de uma forma clínica localizada e não contagiosa a uma forma difusa e contagiosa; entre esses extremos, encontram-se as formas intermediárias, que denotam os diferentes graus de resposta do hospedeiro ao bacilo ${ }^{5}$.

O diagnóstico é estabelecido pela classificação operacional dos casos. A mesma toma por base o número das lesões na pele, até cinco ou mais de cinco lesões, classificando, respectivamente, o caso em paucibacilar (PB) e multibacilar (MB). Quando realizada a baciloscopia com resultado positivo, O caso é classificado como $M B$ independentemente do número de lesões. A negatividade do exame não exclui o diagnóstico de hanseníase e nem classifica o caso como PB. A classificação operacional direciona - esquema do tratamento poliquimioterápico a ser estabelecido para o paciente ${ }^{6}$.

A adolescência tem como característica ser uma fase de mudanças e de adaptações físicas e psicológicas. Devido a isso, a hanseníase pode afetar a construção da vida de um adolescente, com alterações que podem ir desde a evasão escolar até a depreciação da autoimagem ${ }^{7}$.

$\bigcirc$ presente trabalho visou analisar a tendência da taxa de detecção da hanseníase em jovens nas Regiões de Integração do Estado do Pará, no período de 2005 a 2014.

\section{MATERIAIS E MÉTODOS}

Foi realizado um estudo ecológico exploratório, com base em dados coletados das fichas do Sistema de
Informação de Agravos de Notificação (SINAN), o qual é gerenciado pelo Departamento de Epidemiologia da Secretaria de Estado de Saúde Pública do Pará (SESPA), iniciado após aprovação, em 28 de maio de 2015, do Comitê de Ética em Pesquisa em Seres Humanos do Centro Universitário do Estado do Pará, sob o processo de $\mathrm{n}^{\circ} 1.108 .320$.

Os dados coletados foram referentes aos casos novos de hanseníase em jovens ${ }^{8}$ registrados no SINAN e ocorridos no estado do Pará, no período de 2005 a 2014. Foram excluídos os registros de jovens não residentes no estado do Pará, aqueles com idade e/ ou município de residência ignorados, os registros de pacientes fora da faixa etária do estudo, assim como os registros com mais de 50\% das variáveis eleitas para o estudo sem preenchimento.

Para a descrição do perfil sociodemográfico do jovem com hanseníase (pacientes com idade entre 10 e 19 anos), foram analisadas as seguintes variáveis constantes na ficha do SINAN: sexo, raça/cor, idade, escolaridade, gravidez e município de residência. Os aspectos clínico e epidemiológico tiveram como variáveis de análise o ano do diagnóstico da doença, a forma clínica e a classificação operacional.

○estudo foi realizado utilizando-se a divisão regional estabelecida pelo estado do Pará9 ${ }^{9}$, a qual agrupa os 144 municípios constituintes em 12 Regiões de Integração, a saber: Araguaia (15), Baixo Amazonas (13), Rio Caeté (15), Rio Capim (16), Carajás (12), Guamá (18), Lago Tucuruí (sete), Marajó (16), Metropolitana (cinco), Tapajós (seis), Tocantins (11) e Xingu (10).

Os dados foram ajustados por idade, tanto entre as regiões como intrarregionalmente, e não foram encontradas diferenças significantes na distribuição proporcional das diferentes populações de cada município e das regiões ao longo da série histórica analisada. Após o ajuste, os dados foram apresentados sob a forma de tabelas e gráficos e analisados com - auxílio do programa BioEstat v5.310, utilizando-se a estatística descritiva e o teste qui-quadrado de aderência. Foram consideradas como diferenças estatisticamente significantes aquelas com $p<0,05$.

\section{RESULTADOS}

No período de 2005 a 2014, foram notificados, no estado do Pará, 48.651 casos novos de hanseníase. Do total analisado, 7.608 (15,64\%) ocorreram em jovens na faixa etária de 10 a 19 anos, com uma média anual de 760,8 casos novos. Fizeram parte deste estudo 7.606 notificações, pois, em duas dessas, o município teve registro ignorado.

As regiões Carajás (14,2\%) e Metropolitana (14,1\%) foram as que apresentaram as maiores proporções de casos novos de hanseníase em jovens no estado do Pará durante o período do estudo (Tabela 1). No entanto, o maior risco para a ocorrência de novos casos foi observado nas regiões Metropolitana (389,0/100.000), Lago Tucuruí (259,2/100.000) e Carajás (196,7/100.000). 
Tabela 1 - Distribuição dos casos novos e da taxa de detecção cumulativa de hanseníase em jovens segundo as Regiões de Integração do estado do Pará, Brasil, no período de 2005 a 2014

\begin{tabular}{lcccc}
\hline $\begin{array}{l}\text { Regiões de } \\
\text { Integração }\end{array}$ & Casos novos & $\%$ & População* $^{*}$ & $\begin{array}{c}\text { Taxa de } \\
\text { detecção }\end{array}$ \\
\hline Carajás & 1.082 & 14,2 & 550.118 & 196,7 \\
Metropolitana & 1.070 & 14,1 & 275.069 & 389,0 \\
Lago Tucuruí & 914 & 12,0 & 352.560 & 259,2 \\
Rio Capim & 872 & 11,5 & 586.795 & 148,6 \\
Tocantins & 868 & 11,4 & 720.474 & 120,5 \\
Araguaia & 820 & 10,8 & 454.580 & 180,4 \\
Xingu & 544 & 7,1 & 338.139 & 160,9 \\
Marajó & 429 & 5,6 & 477.492 & 89,8 \\
Guamá & 341 & 4,5 & 544.192 & 62,7 \\
Tapajós & 248 & 3,3 & 236.758 & 104,7 \\
Rio Caeté & 226 & 3,0 & 461.610 & 49,0 \\
Baixo & 192 & 2,5 & 669.129 & 28,7 \\
Amazonas & & & & 134,2 \\
Total do & 7.606 & 100,0 & 5.666 .916 & \multicolumn{2}{c}{ Fonte: SINAN-SESPA. } \\
Estado & & & &
\end{tabular}

* População (10 a 19 anos de idade) média para o período; † Por 100.000 pessoas na faixa etária de 10 a 19 anos.

Na tabela 2, observa-se que a maior redução no número de casos novos ocorreu na região Araguaia (69,5\%), seguida de Lago Tucuruí (67,9\%) e Baixo Amazonas (65,6\%). Abaixo do valor de redução observado para o estado do Pará (54,7\%), encontram-se as regiões Tocantins, Rio Capim, Carajás, Rio Caeté e Marajó, sendo essa última a região com a menor redução na taxa de detecção $(16,5 \%)$.

Tabela 2 - Redução da taxa de detecção* de hanseníase em jovens entre os anos de 2005 e 2014, segundo as Regiões de Integração do estado do Pará, Brasil

\begin{tabular}{lcccc}
\hline \multicolumn{1}{c}{$\begin{array}{l}\text { Regiões de } \\
\text { Integração }\end{array}$} & 2005 & 2014 & \multicolumn{2}{c}{ Redução } \\
\hline Araguaia & 39,0 & 11,9 & 27,1 & 69,5 \\
Lago Tucuruí & 47,3 & 15,2 & 32,1 & 67,9 \\
Baixo Amazonas & 4,5 & 1,6 & 2,9 & 65,6 \\
Tapajós & 21,7 & 7,8 & 13,9 & 64,1 \\
Metropolitana & 8,6 & 3,1 & 5,5 & 63,9 \\
Guamá & 13,0 & 4,8 & 8,2 & 63,1 \\
Xingu & 30,1 & 13,5 & 16,6 & 55,1 \\
Tocantins & 15,8 & 7,8 & 8,0 & 50,6 \\
Rio Capim & 20,6 & 10,6 & 10,0 & 48,5 \\
Carajás & 28,4 & 15,6 & 12,8 & 45,1 \\
Rio Caeté & 5,5 & 4,0 & 1,5 & 27,3 \\
Marajó & 10,3 & 8,6 & 1,7 & 16,5 \\
Total do Estado & 16,1 & 7,3 & 8,8 & 54,7 \\
\hline & & & Fonte: SINAN-SESPA.
\end{tabular}

comportamento da taxa de detecção anual nas Regiões, com redução da taxa de detecção acima da redução observada para o Estado, é visualizado na figura 1A. Na figura 1B, encontram-se as Regiões com valores de redução da taxa de detecção de hanseníase na população estudada abaixo da taxa do Estado $(54,7 \%)$.

Tanto para o Estado quanto para as Regiões de Integração, o perfil demográfico dos casos (Tabela 3) consistiu em predomínio do gênero masculino $(53,2 \%)$ e da faixa etária entre 15 e 19 anos (53,1\%), sem diferença estatisticamente significante. No Estado, a média de idade foi de 14,7 ( $\pm 2,8)$; a média inferior foi observada nas regiões Araguaia (14,5 $\pm 2,7)$ e Marajó $(14,4 \pm 2,7)$; e a média superior, nas regiões Carajás (14,6 \pm 2,8), Metropolitana (15,1 \pm 2,9), Tocantins (14,8 $\pm 2,9)$, Rio Capim $(14,8 \pm 2,8)$ e Rio Caeté $(14,8 \pm 2,9)$.

Tabela 3 - Perfil sociodemográfico dos jovens portadores de hanseníase no estado do Pará, Brasil, de 2005 a 2014

\begin{tabular}{lccc}
\hline \multicolumn{1}{c}{ Características } & Casos novos & $\%$ & $p$ \\
\hline Gênero & & & \\
Masculino & 4.046 & 53,2 & 0,5222 \\
Feminino & 3.560 & 46,8 &
\end{tabular}

Faixa etária

$\begin{array}{lll}10 \text { a } 14 \text { anos } & 3.570 & 46,9 \\ 15 \text { a } 19 \text { anos } & 4.036 & 53,1\end{array}$

0,6087

Raça/Cor

$\begin{array}{lrr}\text { Parda } & 5.305 & 69,7 \\ \text { Branca } & 1.039 & 13,7 \\ \text { Amarela } & 108 & 1,4 \\ \text { Preta } & 949 & 12,5 \\ \text { Indígena } & 46 & 0,6 \\ \text { Ignorado } & 159 & 2,1\end{array}$

Escolaridade

Analfabeto

$79 \quad 1,0$

Fundamental incompleto

$5.218 \quad 68,6$

Fundamental completo

333

4,4

Médio incompleto

951

12,5

Médio completo

158

2,1

Superior incompleto

0,3

Ignorado

23

10,1

Gravidez

\begin{tabular}{lrrrr}
$1^{\circ}$ trimestre & 16 & 0,4 & \\
$2^{\circ}$ trimestre & 30 & 0,8 & \\
$3^{\circ}$ trimestre & 15 & 0,4 & $<0,0001$ \\
Ignorado & 16 & 0,4 & \\
Não grávida & 3.483 & 97,8 & \\
\hline
\end{tabular}

\section{0,6}

2,1

, 0

6

$<0,0001$

2,1


A

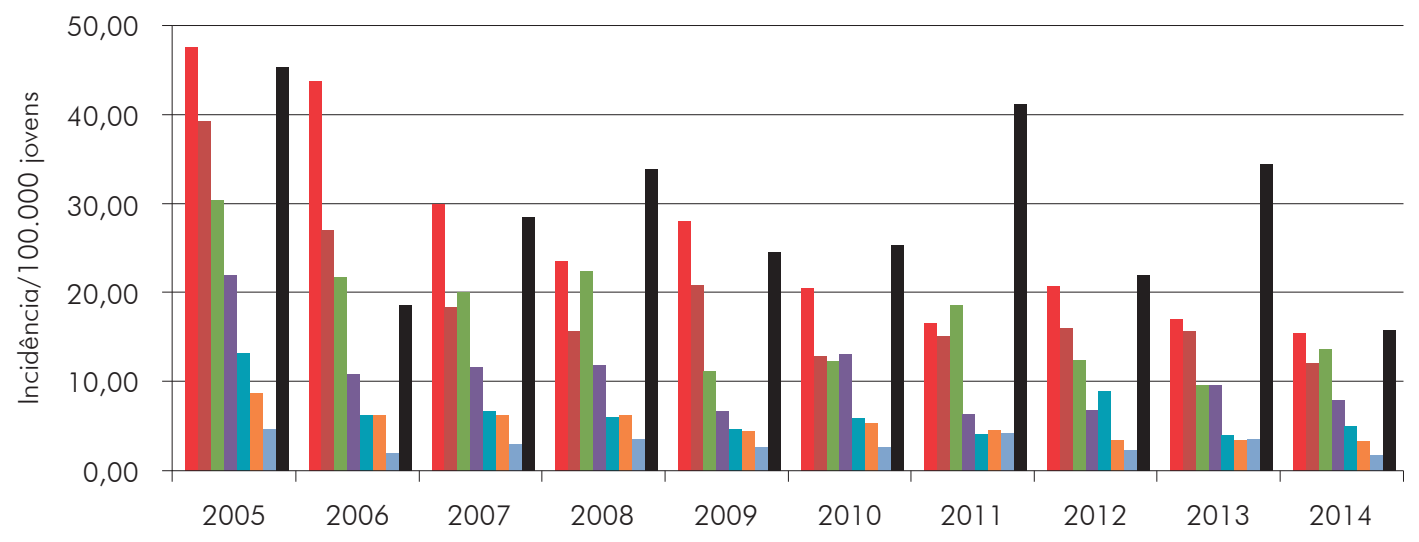

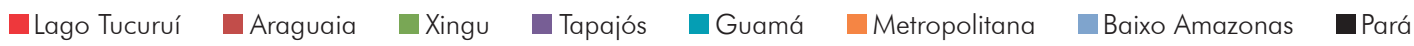

B

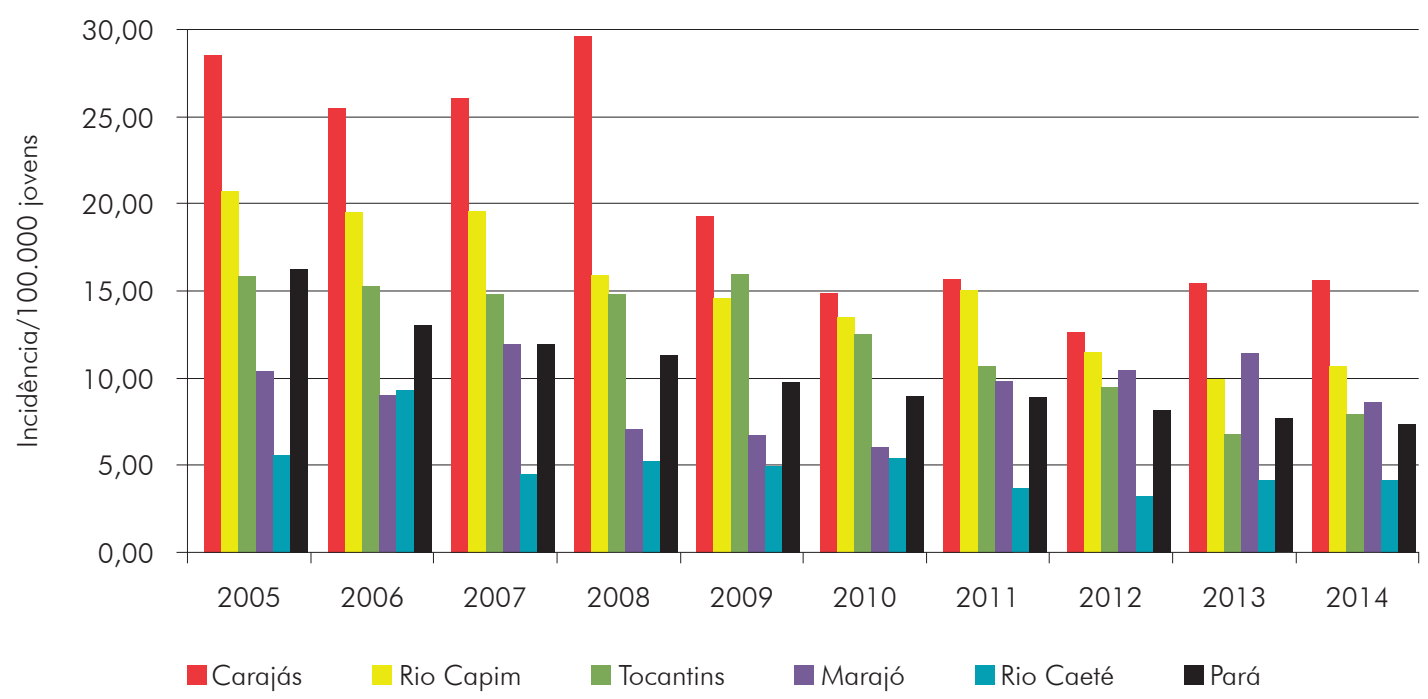

Fonte: SINAN-SESPA. * Por 100.000 pessoas na faixa etária de 10 a 19 anos; ${ }^{\dagger}$ Regiões de Integração com valores de redução da taxa de detecção de hanseníase acima do valor de redução para o estado do Pará $(54,78 \%)$.

Figura 1 - Tendência da taxa de detecção* de hanseníase em jovens segundo algumas Regiões de Integração do estado do Parát, Brasil, no período de 2005 a 2014

A variável raça/cor parda, em relação às demais, apresentou diferença significante $(p<0,0001)$ em todas as Regiões e no Estado. As regiões Rio Capim (65,4\%), Tapajós (68,2\%), Lago Tucuruí $(67,2 \%)$, Carajás $(67,7 \%)$ e Araguaia (63,4\%) apresentaram proporções inferiores a do Estado $(69,7 \%)$.

No Pará, houve predominância significante das proporções de jovens com hanseníase com ensino fundamental incompleto. Proporções superiores à média do Estado $(69,5 \%)$ foram observadas nas regiões Rio Capim (70,7\%), Tapajós (70,9\%), Marajó (77,8\%), Lago Tucuruí $(75,6 \%)$ e Carajás $(74,6 \%)$. Observou-se para - Estado a proporção de 2,1\% de casos com ensino médio completo; nas regiões Carajás, Metropolitana e Rio Caeté, essas proporções foram respectivamente de 2,5\%, 3,5\% e 3,2\%. A região Rio Capim apresentou proporção semelhante à do Estado.
A maior proporção observada, em todo o Estado e suas regiões, foi de não grávidas $(97,8 \%)$; no entanto, foram encontrados registros de jovens grávidas em diferentes trimestres de gravidez, assim como com idade gestacional ignorada. Entre as que possuíam trimestre gestacional definido, predominou, no Estado $(0,8 \%)$, o segundo trimestre gestacional. Nas regiões Rio Caeté $(1,1 \%)$, Xingu (3,3\%), Carajás $(0,93 \%)$, Baixo Amazonas $(1,1 \%)$ e Araguaia $(1,1 \%)$, foram encontrados índices de jovens no segundo trimestre gestacional superiores aos do Estado.

A forma clínica indeterminada foi a mais prevalente (Tabela 4), com diferença estatisticamente significante em relação às demais formas em cada Região de Integração ( $p<0,05)$, sendo que, em algumas regiões, os números foram superiores aos do Estado $(35,9 \%)$. São elas: Araguaia (41,6\%), Baixo Amazonas (38,0\%), Lago Tucuruí $(47,4 \%)$, Rio Capim $(41,9 \%)$ e 
Xingu (43,4\%). A forma clínica dimorfa foi a segunda mais prevalente, com as regiões Carajás (34,4\%), Guamá (34,6\%), Marajó (35,7\%), Metropolitana $(37,8 \%)$ e Tapajós $(37,5 \%)$ apresentando percentuais superiores aos do Estado (31,0\%). Houve predominância da forma clínica dimorfa em quatro (Carajás, Marajó, Metropolitana e Tapajós) das 12 Regiões do Estado.

(continua)

Tabela 4 - Distribuição dos casos novos de hanseníase segundo a forma clínica e classificação operacional nas Regiões de Integração do estado do Pará, Brasil, de 2005 a 2014

\begin{tabular}{|c|c|c|c|c|c|c|}
\hline \multirow{2}{*}{$\begin{array}{l}\text { Regiões de } \\
\text { Integração }\end{array}$} & \multirow{2}{*}{ Forma clínica } & \multicolumn{2}{|c|}{ Classificação operacional } & \multirow{2}{*}{ Total } & \multirow{2}{*}{$\%$} & \multirow{2}{*}{$\mathrm{p}$} \\
\hline & & PB & MB & & & \\
\hline \multirow{7}{*}{ Araguaia } & Indeterminada & 341 & - & 341 & 41,6 & \\
\hline & Tuberculoide & 107 & - & 107 & 13,0 & \\
\hline & Dimorfa & - & 198 & 198 & 24,2 & 0,0004 \\
\hline & Virchowiana & - & 60 & 60 & 7,3 & \\
\hline & Não classificada & 49 & 65 & 114 & 13,9 & \\
\hline & Total & 497 & 323 & 820 & 100,0 & 0,0434 \\
\hline & $\%$ & 60,6 & 39,4 & $8 \angle 0$ & 100,0 & 0,0434 \\
\hline \multirow{7}{*}{ Baixo Amazonas } & Indeterminada & 73 & - & 73 & 38,0 & \\
\hline & Tuberculoide & 34 & - & 34 & 17,7 & \\
\hline & Dimorfa & - & 45 & 45 & 23,5 & 0,0157 \\
\hline & Virchowiana & - & 25 & 25 & 13,0 & \\
\hline & Não classificada & 7 & 8 & 15 & 7,8 & \\
\hline & Total & 114 & 78 & 102 & 1000 & 0075 \\
\hline & $\%$ & 59,4 & 40,6 & 192 & 100,0 & ( \\
\hline \multirow{7}{*}{ Carajás } & Indeterminada & 362 & - & 362 & 33,5 & \\
\hline & Tuberculoide & 149 & - & 149 & 13,8 & \\
\hline & Dimorfa & - & 372 & 372 & 34,4 & 0,0069 \\
\hline & Virchowiana & - & 60 & 60 & 5,5 & \\
\hline & Não classificada & 50 & 89 & 139 & 12,8 & \\
\hline & Total & 561 & 521 & & & 07940 \\
\hline & $\%$ & 51,8 & 48,2 & 1.082 & 100,0 & 0,1949 \\
\hline \multirow{7}{*}{ Guamá } & Indeterminada & 123 & - & 123 & 36,1 & \\
\hline & Tuberculoide & 32 & - & 32 & 9,4 & \\
\hline & Dimorfa & - & 118 & 118 & 34,6 & 0,0002 \\
\hline & Virchowiana & - & 28 & 28 & 8,2 & \\
\hline & Não classificada & 12 & 28 & 40 & 11,7 & \\
\hline & Total & 167 & 174 & & & \\
\hline & $\%$ & 49,0 & 51,0 & 341 & 100,0 & 0,9203 \\
\hline \multirow{7}{*}{ Lago Tucuruí } & Indeterminada & 433 & - & 433 & 47,4 & \\
\hline & Tuberculoide & 123 & - & 123 & 13,5 & \\
\hline & Dimorfa & - & 263 & 263 & 28,8 & $<0,0001$ \\
\hline & Virchowiana & - & 37 & 37 & 4,0 & \\
\hline & Não classificada & 26 & 32 & 58 & 6,3 & \\
\hline & Total & 582 & 332 & 914 & 1000 & 0.0083 \\
\hline & $\%$ & 63,7 & 36,3 & 914 & & \\
\hline \multirow{7}{*}{ Marajó } & Indeterminada & 123 & - & 123 & 28,7 & \\
\hline & Tuberculoide & 65 & - & 65 & 15,1 & \\
\hline & Dimorfa & - & 153 & 153 & 35,7 & $<0,0001$ \\
\hline & Virchowiana & - & 63 & 63 & 14,7 & \\
\hline & Não classificada & 9 & 16 & 25 & 5,8 & \\
\hline & Total & 197 & 232 & 429 & 100,0 & 0,4715 \\
\hline & $\%$ & 45,9 & 54,1 & 429 & 100,0 & $0,4 / 15$ \\
\hline \multirow{7}{*}{ Metropolitana } & Indeterminada & 234 & - & 234 & 21,9 & \\
\hline & Tuberculoide & 260 & - & 260 & 24,3 & \\
\hline & Dimorfa & - & 405 & 405 & 37,8 & 0,0708 \\
\hline & Virchowiana & - & 153 & 153 & 14,3 & \\
\hline & Não classificada & 10 & 8 & 18 & 1,7 & \\
\hline & Total & 504 & 566 & م70 & ח 1 & 06312 \\
\hline & $\%$ & 47,1 & 52,9 & $1.0 / 0$ & 100,0 & 0,6312 \\
\hline
\end{tabular}


Tabela 4 - Distribuição dos casos novos de hanseníase, segundo a forma clínica e classificação operacional nas regiões de integração do Estado do Pará, Brasil, de 2005 a 2014

\begin{tabular}{|c|c|c|c|c|c|c|}
\hline \multirow{2}{*}{$\begin{array}{l}\text { Regiões de } \\
\text { Integração }\end{array}$} & \multirow{2}{*}{ Forma clínica } & \multicolumn{2}{|c|}{ Classificação operacional } & \multirow{2}{*}{ Total } & \multirow{2}{*}{$\%$} & \multirow{2}{*}{$p$} \\
\hline & & PB & $M B$ & & & \\
\hline \multirow{7}{*}{ Rio Caeté } & Indeterminada & 70 & - & 70 & 31,0 & \multirow{7}{*}{0,0234} \\
\hline & Tuberculoide & 21 & - & 21 & 9,3 & \\
\hline & Dimorfa & - & 65 & 65 & 28,7 & \\
\hline & Virchowiana & - & 39 & 39 & 17,3 & \\
\hline & Não classificada & 13 & 18 & 31 & 13,7 & \\
\hline & Total & 104 & 122 & \multirow{2}{*}{226} & \multirow{2}{*}{100,0} & \\
\hline & $\%$ & 46,0 & 54,0 & & & \\
\hline \multirow{7}{*}{ Rio Capim } & Indeterminada & 365 & - & 365 & 41,9 & \multirow{7}{*}{0,0087} \\
\hline & Tuberculoide & 165 & - & 165 & 18,9 & \\
\hline & Dimorfa & - & 228 & 228 & 26,1 & \\
\hline & Virchowiana & - & 67 & 67 & 7,7 & \\
\hline & Não classificada & 17 & 30 & 47 & 5,4 & \\
\hline & Total & 547 & 325 & 872 & 1000 & \\
\hline & $\%$ & 62,7 & 37,3 & $8 / 2$ & 100,0 & \\
\hline \multirow{7}{*}{ Tapajós } & Indeterminada & 76 & - & 76 & 30,6 & \multirow{7}{*}{0,0016} \\
\hline & Tuberculoide & 30 & - & 30 & 12,1 & \\
\hline & Dimorfa & - & 93 & 93 & 37,5 & \\
\hline & Virchowiana & - & 22 & 22 & 8,9 & \\
\hline & Não classificada & 17 & 10 & 27 & 10,9 & \\
\hline & Total & 123 & 125 & 248 & 1000 & \\
\hline & $\%$ & 49,6 & 50,4 & 248 & 100,0 & \\
\hline \multirow{7}{*}{ Tocantins } & Indeterminada & 296 & - & 296 & 34,1 & \multirow{7}{*}{0,1676} \\
\hline & Tuberculoide & 140 & - & 140 & 16,1 & \\
\hline & Dimorfa & - & 245 & 245 & 28,2 & \\
\hline & Virchowiana & - & 71 & 71 & 8,2 & \\
\hline & Não classificada & 62 & 54 & 116 & 13,4 & \\
\hline & Total & 498 & 370 & 868 & م 100 & \\
\hline & $\%$ & 57,4 & 42,6 & & & \\
\hline \multirow{7}{*}{ Xingu } & Indeterminada & 236 & - & 236 & 43,4 & \multirow{7}{*}{0,0001} \\
\hline & Tuberculoide & 62 & - & 62 & 11,4 & \\
\hline & Dimorfa & - & 169 & 169 & 31,1 & \\
\hline & Virchowiana & - & 32 & 32 & 5,9 & \\
\hline & Não classificada & 37 & 8 & 45 & 8,2 & \\
\hline & Total & 335 & 209 & 544 & 100,0 & \\
\hline & $\%$ & 61,6 & 38,4 & 544 & 100,0 & \\
\hline \multirow{7}{*}{ Estado do Pará } & Indeterminada & 2.732 & - & 2.732 & 35,9 & \multirow{5}{*}{0,0170} \\
\hline & Tuberculoide & 1.188 & - & 1.188 & 15,6 & \\
\hline & Dimorfa & - & 2.354 & 2.354 & 31,0 & \\
\hline & Virchowiana & - & 657 & 657 & 8,6 & \\
\hline & Não classificada & 309 & 366 & 675 & 8,9 & \\
\hline & Total & 4.229 & 3.377 & 7606 & 0 & 3077 \\
\hline & $\%$ & 55,6 & 44,4 & & & \\
\hline
\end{tabular}

Quanto à classificação operacional (Tabela 4), a forma PB foi a mais prevalente no Estado (55,6\%), sem diferença estatisticamente significante em relação à forma MB. Apenas a região Carajás (51,8\%) apresentou índice inferior ao do Estado, enquanto que as regiões Araguaia (60,6\%), Baixo Amazonas $(59,45)$, Lago Tucuruí (63,7\%), Rio Capim (62,7\%), Tocantins
$(57,4 \%)$ e Xingu $(61,6 \%)$ mostraram valores superiores. Em cinco Regiões (Guamá, Marajó, Metropolitana, Rio Caeté e Tapajós), predominou a forma MB.

Do total de casos novos de hanseníase na população de jovens de 10 a 19 anos de idade, $39,6 \%$ foram notificados nas formas clínicas dimorfa e virchowiana (Tabela 4). 
Comparando-se os percentuais da forma indeterminada, dentre as PB (2.732/4.229), com os da forma dimorfa, dentre as MB (2.354/3.377), essa detém $69,7 \%$, enquanto a indeterminada detém $64,6 \%$ (Tabela 4).

\section{DISCUSSÃO}

Brasil apresenta as maiores taxas de detecção de casos novos de hanseníase dentre os países do continente americano e, no mundo, fica atrás apenas da Índia. Em 2014, foram notificados 31.064 casos novos no Brasil ${ }^{3}$. No ano de 2015, a maior concentração desses casos deu-se nas Regiões Nordeste, Centro-Oeste e Norte do Brasil ${ }^{1}$.

A análise dos casos novos de hanseníase no estado do Pará mostrou que as regiões Carajás e Metropolitana foram as que contribuíram com as maiores proporções da doença em jovens. Esse achado condiz com um estudo realizado no período de 2004 a 2006, também no Pará, o qual demonstrou que o maior número de casos novos ocorreu nos municípios de Belém e Ananindeua (região Metropolitana), além dos municípios de Marabá e Parauapebas (região (arajás) $)^{12}$.

Foram observadas, nas regiões Metropolitana, Lago Tucuruí e Carajás, as maiores taxas de detecção de hanseníase. Segundo o Ministério da Saúde, dos 253 municípios brasileiros com casos novos diagnosticados em 2010, o Pará apresentou 42 (16,6\%) municípios, - que representa $29,2 \%$ do total de municípios paraenses. Esses municípios estão distribuídos nas Regiões de Integração, com exceção da região Rio Caeté. Embora tais municípios constituam áreas geográficas de risco no Pará, os que possuem maiores taxas de detecção no plano integrado de ações estratégicas do Ministério da Saúde, no período de 2001 a 2010, foram Jacareacanga (região Tapajós), Mãe do Rio (região Rio Capim) e Jacundá (região Lago Tucuruí $^{13}$. Embora o Ministério da Saúde não ressalte a faixa etária aqui trabalhada, deve ser enfatizado que as estratégias para alcançar as metas de eliminação precisam ser desenvolvidas em todas as faixas etárias, principalmente nas idades menores, face às sequelas psicológicas.

O Pará é classificado, segundo os parâmetros da OMS, como de alta endemicidade para hanseníase. Por isso, 42 municípios paraenses são considerados prioritários para monitoramento das ações de controle da doença, estando inseridos nas seguintes Regiões de Integração: Araguaia (quatro), Baixo Amazonas (um), Carajás (três), Guamá (um), Lago Tucuruí (cinco), Marajó (dois), Metropolitana (três), Rio Capim (oito), Tapajós (três), Tocantins (nove) e Xingu (três) ${ }^{13}$.

A prevalência da hanseníase vem caindo ano após ano no Brasil, talvez como resultado da maior adesão ao esquema de tratamento poliquimioterápico e seu consequente sucesso. $\bigcirc$ Pará, no entanto, assim como os outros estados da Região Norte, tem tido anualmente acréscimos de registros da incidência da enfermidade, o que pode representar os resultados do investimento realizado, a partir de 2001, na capacitação de profissionais de saúde da atenção primária para a detecção da doença ${ }^{12}$.

Na descrição do perfil epidemiológico nas Regiões de Integração do Pará, foram observados alguns padrões, tais como: o predomínio do sexo masculino, a raça/cor parda e a baixa escolaridade. $\bigcirc$ achado quanto ao sexo, na faixa etária de 10 a 19 anos, foi semelhante ao observado em um estudo realizado em Fortaleza, estado do Ceará ${ }^{14}$, e em outro no estado do Maranhão ${ }^{15}$, em que ambos abordaram a faixa etária de 10 a 19 anos; além de outra pesquisa realizada no estado do Piauíl $^{6}$, que avaliou as taxas de detecção e prevalência na população geral e em menores de 15 anos de idade. Em geral, os coeficientes de detecção de casos de hanseníase são maiores entre os homens, por ser um grupo com maior risco de exposição; entretanto, em uma pesquisa feita na cidade de Sobral, no Ceará$^{7}$, em que foi utilizada a mesma faixa etária, identificou-se maior ocorrência no sexo feminino.

A raça/cor parda foi predominante em todo o Pará e suas regiões. Esses dados convergiram com um estudo descritivo realizado em Ananindeua, Pará ${ }^{17}$, com estudantes do Ensino Fundamental e também com um estudo realizado em Jaguaré, estado do Espírito Santo $^{18}$, que estudou as idades de 6 a 84 anos. Esse achado talvez reproduza o processo histórico de colonização, miscigenação, movimentos migratórios, dinâmica de ocupação territorial e organização espacial $^{19}$.

A baixa escolaridade observada nesta pesquisa também foi relatada em um estudo ecológico realizado entre 2003 e 2013, em Igarapé-Açu, Pará20, o qual abordou a faixa etária de menores de 15 anos; assim como em uma pesquisa feita em Sobral, no Ceará ${ }^{7}$. Esses achados denotam que as condições de vida da população e o nível de escolaridade influenciam a prevalência de doenças endêmicas como a hanseníase ${ }^{21}$.

Um estudo retrospectivo feito na região Carajás ${ }^{22}$ constatou que o número de mulheres grávidas afetadas pela hanseníase é muito menor do que na população em geral, corroborando o encontrado, que foi de aproximadamente 2,2\% de adolescentes grávidas, haja vista que o Sistema de Informação de Nascidos Vivos (SINASC) registrou 406.516 nascimentos nessa faixa etária no período estudado, o que pode expressar $25,8 \%$ de gravidez nessa população residente no Estado ${ }^{23}$

No entanto, um estudo realizado em Fortaleza, em $2011^{24}$, constatou que a maioria dos sinais de hanseníase entre grávidas e/ou lactantes ocorreu durante o último trimestre de gravidez e nos três meses após o parto, divergindo dos achados deste estudo. Embora tenha sido associada a graves consequências clínicas, a relação entre hanseníase e gravidez ainda é mal compreendida, visto que, no Brasil, os dados sobre a hanseníase durante a gravidez só se tornaram confiáveis e disponíveis a partir de 2007 
no SINAN, o que reduz e limita a análise desses dados $^{22}$.

A classificação operacional predominante nas Regiões de Integração do estado do Pará foi a PB. Resultado semelhante foi também encontrado em uma pesquisa no Piauí, entre 2003 e 2008 ${ }^{15}$, e outra no Pará, que avaliou casos novos de hanseníase notificados no período de 2003 a 2013, em menores de 15 anos, em uma área de antiga colônia de portadores da doença ${ }^{20}$. Entretanto, foram encontrados na literatura resultados discordantes, como o observado no estudo feito em Maricá, estado do Rio de Janeiro ${ }^{25}$, que abordou essa variável distribuindo os casos em maiores e menores de 15 anos; bem como em um estudo realizado em Fortaleza ${ }^{14}$, no qual a forma MB prevaleceu entre os jovens de 10 a 19 anos de idade.

Embora o achado da maioria de casos novos, na forma clínica intederminada dentre os PB, possa sugerir que o diagnóstico precoce esteja ocorrendo em todo - Estado, observou-se a ocorrência de 44,4\% de casos novos $M B$, o que se considera uma proporção elevada e não esperada para essa faixa etária. Isso se justificaria pelo atraso no diagnóstico da doença, o que influencia a manutenção da cadeia epidemiológica, visto que os casos $\mathrm{MB}$ refletem o caráter endêmico da região estudada, fazendo com que esses pacientes representem uma fonte de infecção ${ }^{26}$.

A forma clínica indeterminada, seguida da dimorfa, foram os registros mais encontrados. A forma indeterminada, que pode evoluir para as formas polarizadas ou para cura, também se mostrou prevalente em uma pesquisa realizada no Piauí ${ }^{16}$. Divergindo desse resultado, estudos realizados em jovens, no Distrito Federal $^{27}$ e em Fortaleza ${ }^{14}$, tiveram como achados, respectivamente, a predominância da forma tuberculoide e da forma dimorfa.

- predomínio da forma clínica dimorfa dentre os casos MB, em proporção superior à indeterminada dentre os casos PB, assim como o registro de casos com forma clínica ignorada, requer maior atenção dos serviços de saúde, uma vez que deve ser priorizada a prevenção contra as sequelas determinadas pela doença quando ocorrer o diagnóstico tardio, além de controlar a endemia ${ }^{6}$.

A relevância epidemiológica e clínica dessa constatação é que a forma dimorfa tem um grande poder de transmissibilidade e um alto índice de incapacidade residual. Por isso, esses dados devem ser discutidos entre os profissionais de saúde, como estratégia de sensibilização para diagnóstico e abordagem precoce da doença ${ }^{19}$.

Mesmo que o presente estudo possua vieses de análise comparativa com a literatura, devido à exiguidade de estudos que abordem a faixa etária de jovens, a fragilidade no diagnóstico demonstrado pelos registros ignorados suscita mais efetividade do programa de controle da hanseníase. Ainda se fazem necessárias estratégias educativas de saúde para informar a população quanto aos sinais e sintomas, consequências clínicas e tratamento da hanseníase.

\section{CONCLUSÃO}

Embora em sete Regiões se tenha observado a redução da incidência, há uma estabilidade no Estado nos últimos cinco anos, o que denota a permanência de elevada endemicidade e a carência de ações efetivas de educação em saúde para a população e de comprometimento de recursos humanos em saúde com a meta de redução da hanseníase no Brasil.

Observou-se que houve uma distribuição heterogênea da doença no Estado ao se identificar que duas das Regiões de Integração, Carajás e Metropolitana, detiveram a maior proporção de casos novos e quatro tiveram predomínio da forma clínica dimorfa. Por isso, considera-se que o estado do Pará ainda se encontra distante de uma evolução favorável no controle da hanseníase.

\section{REFERÊNCIAS}

1 Ministério da Saúde (BR). Secretaria de Vigilância em Saúde. Vigilância em Saúde: situação epidemiológica da hanseníase no Brasil, 2008 [Internet]. Brasília: Ministério da Saúde; 2008 [citado 2012 jan 21]. Disponível em: http://bvsms.saude.gov.br/bvs/ publicacoes/vigilancia_saude_situacao_hanseniase. pdf.

2 World Health Organization. Global leprosy situation, beginning of 2008. Wkly Epidemiol Rec. 2008 Aug;83(33):293-300.

3 World Health Organization. Global leprosy update, 2014: need for early case detection. Wkly Epidemiol Rec. 2015 Sep;90(36):461-76.
4 Ministério da Saúde (BR). Secretaria de Vigilância em Saúde. Sistema de Informações de Agravos e Notificações. Taxa de prevalência da hanseníase, estados, Brasil (31/05/2016) [Internet]. Brasília: Ministério da Saúde; 2015 [citado 2016 ago 23]. Disponível em: http://portalarquivos.saude.gov.br/ images/pdf/2016/julho/07/Taxa-de-Preval--nciada-Hansen--ase--estados--Brasil--2015.pdf.

5 Souza CS. Hanseníase: formas clínicas e diagnóstico diferencial. Medicina. 1997 jul-set;30(3):325-34.

6 Ministério da Saúde (BR). Secretaria de Vigilância em Saúde. Departamento de Vigilância das Doenças Transmissíveis. Diretrizes para vigilância, atenção e eliminação da hanseníase como problema de saúde pública. Brasília: Ministério da Saúde; 2016. 60 p. 
7 Ponte KMA, Ximenes Neto FRG. Hanseníase: a realidade do ser adolescente. Rev Bras Enferm. 2005 mai-jun;58(3):296-301.

8 Brasil. Lei n. 8.069 de 13 de julho de 1990. Dispõe sobre o Estatuto da Criança e do Adolescente e dá outras providências [Internet]. Brasília: Presidência da República, Casa Civil; 2015 [citado 2015 abr 22]. Disponível em: http://www.planalto.gov.br/ ccivil 03/leis/l8069.htm.

9 Pará. Decreto Estadual n. 1.066 de 19 de junho de 2008. Dispõe sobre a regionalização do Estado do Pará e dá outras providências [Internet]. Belém: Ministério Público Federal, Procuradoria da República no Pará; 2015 [citado 2015 abr 22]. Disponível em: http://www.prpa.mpf.mp.br/setorial/ biblioteca/legislacao/decreto-estadual-n-1-066-de19-de-junho-de-2008.

10 Ayres M, Ayres Jr M, Ayres DL, Santos AS. BioEstat 5.0: aplicações estatísticas nas áreas das ciências biológicas e médicas. Belém: Mamirauá; 2007. 364 p.

11 Ministério da Saúde (BR). Sistema de Informação de Agravos Notificáveis. Registro ativo: número e percentual, casos novos de hanseníase: número, coeficiente e percentual, faixa etária, classificação operacional, sexo, grau de incapacidade, contatos examinados, por estados e regiões, Brasil, 2015 [Internet]. Brasília: Ministério da Saúde; 2015 [citado 2016 ago 23]. Disponível em: http://portalarquivos. saude.gov.br/images/pdf/2016/julho/07/tabelageral-2015.pdf.

12 Palácios VRCM, Dias RS, Neves DCO. Estudo da situação da hanseníase no Estado do Pará. Rev Para Med. 2010 abr-jun;24(2):49-56.

13 Ministério da Saúde (BR). Secretaria de Vigilância em Saúde. Departamento de Vigilância das Doenças Transmissíveis. Plano integrado de ações estratégicas de eliminação da hanseníase, filariose, esquistossomose e oncocercose como problema de saúde pública, tracoma como causa de cegueira e controle das geohelmintíases: plano de ação 2011 2015. Brasília: Ministério da Saúde; 2012. p. 65-6.

14 Pereira EMSF. Características clínicas da hanseníase em adolescentes em uso de poliquimioterapia [dissertação na Internet]. Fortaleza (CE): Universidade Estadual do Ceará, Centro de Ciências da Saúde; 2007 [citado 2016 ago 23]. Disponível em: http:// www. vece.br/mpsca/index.php/arquivos/doc download/254.

15 Lustosa AA, Nogueira LT, Pedrosa JIS, Teles JBM, Campelo V. The impact of leprosy on healthrelated quality of life. Rev Soc Bras Med Trop. 2011 Sep-Oct;44(5):621-6.

16 Sousa MWG, Silva DC, Carneiro LR, Almino MLBF, Costa ALF. Epidemiological profile of leprosy in the Brazilian state of Piauí between 2003 and 2008. An Bras Dermatol. 2012 May-Jun;87(3):389-95.
17 Sousa BRM, Moraes FHA, Andrade JS, Lobo ES, Macedo EA, Pires CAA, et al. Educação em saúde e busca ativa de casos de hanseníase em uma escola pública em Ananindeua, Pará, Brasil. Rev Bras Med Fam Comunidade. 2013 abr-jun;8(27): 143-9.

18 Santos AS, Castro DS, Falqueto A. Fatores de risco para transmissão da hanseníase. Rev Bras Enferm. 2008 nov;61 no. esp:738-43.

19 Ribeiro Júnior AF, Vieira MA, Caldeira AP. Perfil epidemiológico da hanseníase em uma cidade endêmica no Norte de Minas Gerais. Rev Bras Clin Med. 2012 jul-ago; 10(4):272-7.

20 Franco MCA, Macedo GMM, Menezes BQ, Jucá Neto FOM, Franco ACA, Xavier MB. Perfil de casos e fatores de risco para hanseníase, em menores de quinze anos, em município hiperendêmico da Região Norte do Brasil. Rev Para Med. 2014 out-dez;28(4):29-40.

21 Souza VB, Silva MRF, Silva LMS, Torres RAM, Gomes $\mathrm{KWL}$, Fernandes MC, et al. Perfil epidemiológico dos casos de hanseníase de um centro de saúde da família. Rev Bras Promoç Saude. 2013 mar;26(1):110-6.

22 Palácios VRCM, Bichara CNC, Dias RS, Sousa Júnior AS, Cardoso RF, Barreiros GVN, et al. Analysis of the detection coefficient for the association between leprosy and pregnancy in the integration region of Carajás, State of Pará, Brazil. Rev Soc Bras Med Trop. 2013 Sep-Oct;46(5):650-3.

23 Ministério da Saúde (BR). Departamento de Informática do SUS. Sistema de Informação de Nascimento (SINASC): nascidos vivos - Pará [Internet]. Brasília: Ministério da Saúde; 2016 [citado 2016 ago 23]. Disponível em: http://tabnet.datasus. gov.br/cgi/tabcgi.exe?sinasc/cnv/nvpa.def.

24 Nogueira PSF, Moura ERF, Dias AA, Américo CF, Aguiar LR, Valente MMQP. Characteristics of pregnant and lactating women with leprosy. Rev Soc Bras Med Trop. 2015 Jan-Feb;48(1):96-8.

25 Oliveira JCF, Leão AMM, Britto FVS. Análise do perfil epidemiológico da hanseníase em Maricá, Rio de Janeiro: uma contribuição da enfermagem. Rev Enferm. 2014 nov-dez;22(6):815-21.

26 Corrêa RGCF, Aquino DMC, Caldas AJM, Amaral DKCR, França FS, Mesquita ERRBPL. Epidemiological, clinical, and operational aspects of leprosy patients assisted at a referral service in the state of Maranhão, Brazil. Rev Soc Bras Med Trop. 2012 Jan-Feb;45(1):89-94.

27 Lima MAR, Prata MO, Moreira D. Perfil da hanseníase no Distrito Federal no período de 2000 a 2005. Com Cienc Saude. 2008 abr-jun; 19(2): 163-70. 